\title{
IMPROVEMENT OF TRAFFIC OVERHEAD AND RELIABILITY IN WIRELESS SENSOR NETWORK
}

\author{
G.M. Mashrur-E-Elahi ${ }^{1}$, Din Mohammad Mobin ${ }^{2}$, Md. Anwar Ul Islam Rajib ${ }^{3}$ \\ Department of Computer Science and Engineering, \\ Khulna University of Engineering and Technology, Khulna, Bangladesh \\ ${ }^{1}$ ranju2k4cse_kuet@yahoo.com \\ ${ }^{2}$ mobincsedyahoo.com \\ ${ }^{3}$ rajib0607014@yahoo.com
}

\begin{abstract}
In wireless sensor network reliability and traffic overhead is an important issue. To improve the reliability we must, transmit the data in multiple paths from source node to sink node. Source node is a node which collects data through its sensing devices, finds neighbor nodes and sends message to them; all intermediate nodes, which relay the data through multi-hop communication, have the same configuration as the source nodes. Sink node is a special, single node serving as the target, the message receiver. If we transmit the same data in different path then the network become over loaded. So it is necessary to use data splitting technique in multipath routing protocol. Again if we transmit the data in those paths which are unable to reach to the destination successfully then it is necessary to retransmit the data. So for preventing retransmission or data failing on the network we use such an algorithm to select the multiple paths which are successfully transmitting data from source to sink node. We use energy efficient double routing tree construction algorithm to select the active path for sending data from source to sink with guarantee. First we determine the number of path in the sensor network. To determine the number of path we use the double routing tree construction algorithm. Then we determine the number of node of each path. Then we split the data packet according to the ratio of number of nodes. The path which contains minimum number of nodes we transmit maximum number of data packet in that way. As first we determine the number of reliable path it ensures the reliability of the data sending as well as our algorithm. On the other hand as we using splitting technique it minimizes the traffic overhead on the network. Thus we ensure the reliability as well as decrease the traffic overhead on the network.
\end{abstract}

\section{KEYWORDS}

Source node, sink node, query message, multi path degree, traffic, failing probability

\section{INTRODUCTION}

Wireless Sensor Networks (WSNs) consist of small nodes with sensing, computation, and wireless communications capabilities. Due to recent technological advances, the manufacturing of small and low cost sensors became technically and economically feasible. The sensing electronics measure ambient conditions related to the environment surrounding the sensor and transform them into an electric signal. Processing such a signal reveals some properties about objects located and/or events happening in the vicinity of the sensor. A large number of these disposable sensors can be networked in many applications that require unattended operations. A Wireless Sensor Network (WSN)[1]contains hundreds or thousands of these sensor nodes. These sensors have the ability to communicate either among each other or directly to an external base-station DOI : $10.5121 /$ ijcsit.2012.4305 
(BS). A greater number of sensors allows for sensing over larger geographical regions with greater accuracy. Sensor nodes are usually scattered in a sensor field, which is an area where the sensor nodes are deployed. Sensor nodes coordinate among themselves to produce high-quality information about the physical environment. Each sensor node bases its decisions on its mission, the information it currently has, and its knowledge of its computing, communication, and energy resources. Each of these scattered sensor nodes has the capability to collect and route data either to other sensors or back to an external base station(s). A base-station maybe a fixed node or a mobile node capable of connecting the sensor network to an existing communications infrastructure or to the Internet where a user can have access to the reported data. Networking sensor nodes[1] may have profound effect on the efficiency of many military and civil applications.

Previously there are lots of works have been done on wireless sensor network. Different network protocols like multi-path based routing, negotiation based routing, query based routing, quality of service based routing etc. protocols have been proposed. Among these multi-path routing protocol is widely used for reliable data transfer. An energy efficient multi-path routing protocol has been proposed[4]. The aim of this protocol is efficiently find the multiple paths from a source to a destination costing of minimum energy. But this techniques only provide the reliable paths but does not provide the techniques for reducing traffic overhead for packet data transfer. To overcome the traffic overhead another technique was proposed called data splitting technique[2]. This technique split the data packet and send them using redundant paths. However it reduces the traffic but does not ensure the reliable multi-paths to transmit the split packets into the networks. Now the problem is energy efficient multi-path routing protocol [4] ensure only the reliable multiple paths and data splitting technique proposed in [2] reduces the traffic overhead. First one needs a better technique for reducing traffic and second one requires a better technique for reliable multi-paths. Therefore both the technique requires the other to ensure both reliability and reduced traffic overhead.

In this paper we propose a technique that uses both the energy efficient multi-path routing protocol[4] and data splitting technique proposed in [2] to ensure both multi-path reliability and reduced traffic for packet data transmission. At first we find the reliable multiple paths from a source to a destination using the energy efficient multi-path routing protocol and then split the packets using packet data splitting technique and finally sends that split up packets through the generated multiple paths. Simulation results shows that this techniques ensures both the reliable paths and reduced the traffic overhead simultaneously.

The rest of the paper is organized as follows. Section 2 provides an overview of the related recent research work. Different types of routing protocols are described in Section 3. Section 4 describes different existing multipath routing protocols. Data splitting technique is described in Section 5. Our proposed technique is explained in Section 6. Details discussion about simulation and results in Section 7. Section 8 provides a concluding remark of this.

\section{RELATED WORK}

There are different kind of routing protocols in wireless sensor network to transmit data from source node to sink node. In traditional best-effort routing throughput and average response time are the main concerns. Multipath based routing[2] provide good feedback in data transmission.

Multipath based routing is used to send data from source to destination effectively. Multipath based routing also assures quality of service (QoS). QoS routing[3] is usually performed through resource reservation in a connection-oriented communication in order to meet the QoS 
requirements for each individual connection. While many mechanisms have been proposed for routing QoS constrained real-time multimedia data in wire-based networks, they cannot be directly applied to wireless sensor networks due to the limited resources, such as bandwidth and energy that a sensor node has. On the other hand, a number of protocols have been proposed for QoS routing in wireless ad-hoc networks taking the dynamic nature of the network into account. However, none of these protocols consider energy awareness along with the QoS parameters. Some of the proposed protocols consider the imprecise state information while determining the routes.

There are different types of multi-path protocols have been proposed. Among them energy efficient multi-path routing protocol [4] shows better performance. In this technique different paths have been generated by sending query and search message from source to destination. Also for ensuring reliability and reducing traffic overhead another technique was proposed in [2]. In this technique, for different multi-paths the data packet first split into sub-packets and then sends through the paths and thus control the traffic overhead of the network. Both the above techniques does not ensure traffic overhead and multi-path reliability respectively. In this paper we proposed a technique that combines the above two techniques. We use energy efficient multi-path routing protocol for finding reliable multi-paths and then send data packets using data splitting techniques and thus ensure reduced traffic overhead.

\section{Different TyPes of Routing Protocols}

Routing is the process of selecting paths in a network along which to send network traffic. Routing is performed for many kinds of networks, including the telephone network (Circuit switching), electronic data networks (such as the Internet), transportation networks, and wireless sensor network. Routing schemes differ in their delivery semantics. Different routing schemes are: unicast: delivers a message to a single specified node. Broadcast: delivers a message to all nodes in the network. Multicast: delivers a message to a group of nodes that have expressed interest in receiving the message. Anycast: delivers a message to any one out of a group of nodes, typically the one nearest to the source. Geocast : delivers a message to a geographic area.

We have studied a lot of papers about different routing protocols[2] in wireless sensor network. There are different types of routing protocols in WSN. The routing protocols in WSN is categorized in two different ways. The network structure based protocols and operation based protocols. Some of the network structure based protocols are briefly describe below.

\subsection{Flat networks routing}

In flat networks[2], each node typically plays the same role and sensor nodes collaborate together to perform the sensing task. Due to the large number of such nodes, it is not feasible to assign a global identifier to each node. This consideration has led to data centric routing, where the BS sends queries to certain regions and waits for data from the sensors located in the selected regions. Since data is being requested through queries, attribute-based naming is necessary to specify the properties of data.

\subsection{Hierarchical touting}

Hierarchical or cluster-based routing [2], originally proposed in wire line networks, are wellknown techniques with special advantages related to scalability and efficient communication. As such, the concept of hierarchical routing is also utilized to perform energy-efficient routing in WSNs. In a hierarchical architecture, higher energy nodes can be used to process and send the 
information while low energy nodes can be used to perform the sensing in the proximity of the target. This means that creation of clusters and assigning special tasks to cluster heads can greatly contribute to overall system scalability, lifetime, and energy efficiency. Hierarchical routing is an efficient way to lower energy consumption within a cluster and by performing data aggregation and fusion in order to decrease the number of transmitted messages to the BS.

\subsection{Location based routing protocol}

In this kind of routing [2], sensor nodes are addressed by means of their locations. The distance between neighbouring nodes can be estimated on the basis of incoming signal strengths. Relative coordinates of neighbouring nodes can be obtained by exchanging such information between neighbours. Alternatively, the location of nodes may be available directly by communicating with a satellite, using Global Positioning System (GPS), if nodes are equipped with a small low power GPS receiver. To save energy, some location based schemes demand that nodes should go to sleep if there is no activity. More energy savings can be obtained by having as many sleeping nodes in the network as possible. The problem of designing sleep period schedules for each node in a localized manner was addressed.

In operation based protocol there are several kinds of routing algorithm. Among them we studied only the multipath and quality of service (QoS) routing protocol. Different persons give different types of algorithm for quality of service and multipath routing protocol. Among them the algorithm that is used in WSN they are given below:

\subsection{Quality of service protocol}

In quality of service protocol there are two types of algorithm. They are described below:

\subsubsection{Sequential Assignment Routing}

Sequential Assignment Routing (SAR)[2] is one of the first routing protocols for WSNs that introduces the notion of QoS in the routing decisions. Routing decision in SAR depends on three factors: energy resources, QoS on each path, and the priority level of each packet. To avoid single route failure, a multi-path approach is used and localized path restoration schemes are used. The objective of SAR algorithm is to minimize the average weighted QoS metric throughout the lifetime of the network. Simulation results showed that SAR offers less power consumption than the minimum-energy metric algorithm, which focuses only the energy consumption of each packet without considering its priority. SAR maintains multiple paths from nodes to BS. Although, this ensures fault-tolerance and easy recovery, the protocol suffers from the overhead of maintaining the tables and states at each sensor node especially when the number of nodes is huge.

\subsubsection{Speed protocol}

Another QoS routing protocol[2] for WSNs that provides soft real-time end-to-end guarantees was SPEED, which can provide congestion avoidance when the network is congested. The outing module in SPEED is called Stateless Geographic Non-Deterministic forwarding (SNFG) and works with four other modules at the network layer SPEED maintains a desired delivery peed across sensor networks with a two-tier adaptation included for diverting traffic at the networking layer and locally regulating packets sent to the MAC layer. 


\subsection{Multi-path routing protocol}

There are several different papers which describe the algorithm for multipath protocol[3]. Among them two papers [3] an [4] gives the most efficient way of routing. But they also have some limitation. We describe the algorithm briefly and point out the limitations in next section. In multipath routing protocol discover the multiple path from source to destination using an energy efficient way. Tree construction for multiple path is a dynamic way.

\section{Different Existing Multipath Routing Protocol}

In multipath Routing protocol [3][4], main focus is on finding multiple successful paths from source to destination. For finding all multiple paths from source to destination at first need to construct a tree that contains all nodes between source and sink. In multiple path technique all path from source to destination are used for sending data. In multi path technique they also focus on quality of services. All protocol tries to decrease traffic overhead and increase reliability in multiple paths at data sending time. From all existing multi path routing protocol we choose two multipath routing protocols. They contain some limitation. We analyze their protocol and try to solve their limitation. These two existing multipath routing protocol are shortly describe below.

\subsection{Traffic overhead and reliability in multipath Routing}

In wireless sensor networks (WSN) data produced by one or more sources usually has to be routed through several intermediate nodes to reach the destination. Problems arise when intermediate nodes fail to forward the incoming messages. The reliability of the system can be increased by providing several paths from source to destination and sending the same packet through each of them . Using this technique, the traffic increases significantly. In this paper, we analyze a new mechanism that enables the tradeoff between the amount of traffic and the reliability. In the protocol[3] packet is divided into sub packets and this sub packets are send via multiple paths.

In this paper [3] the authors do not clearly define how it will work in dynamic environment. The authors also do not clearly define how the efficient path will be obtained from source to destination. Authors only clearly define how data will be transmitting by low traffic overhead. For this reason this protocol does not provide a good reliability in data sending from source to sink.

Out of many possible designs for multipath routing protocols, two distinct mechanisms exist: disjoint and braided. Disjoint multipath routing tries to construct alternate paths which are node disjoint with the primary path, and with each other. Thus they are unaffected by failure on the primary path. But those alternate paths could potentially have much longer latency than the primary path and therefore expend significantly more energy than that on the primary path. Braided multipath routing relaxes the requirement for node disjoint, which means alternate paths in a braid are partially overlay with the primary path, not completely node disjoint. 


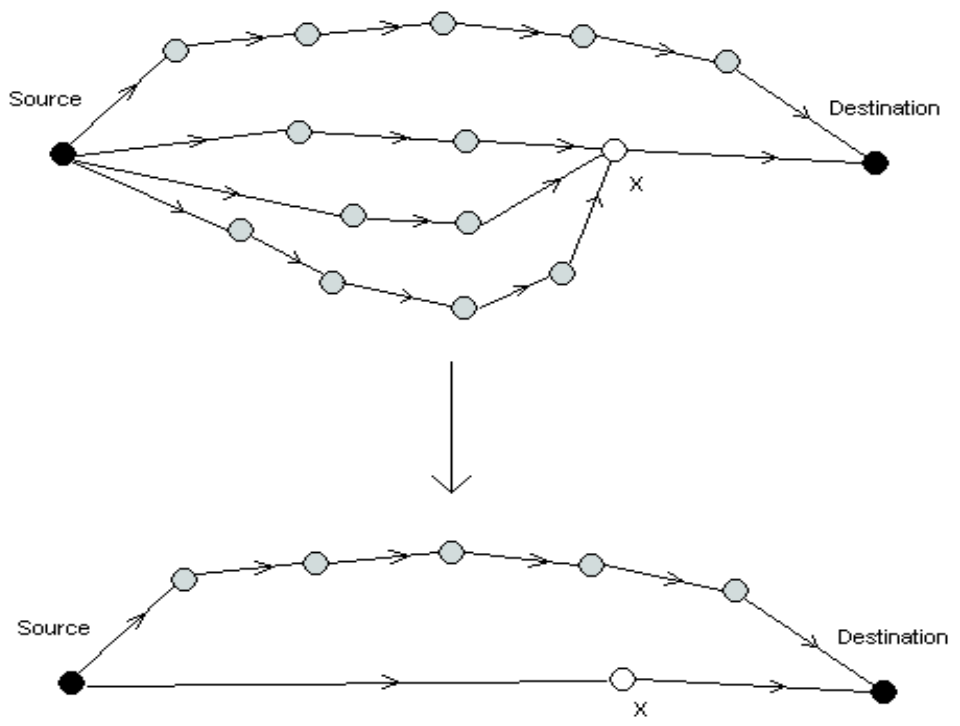

Figure 3. Braided multipath case

In this case, we only consider the disjoint multipath cases[3], because the braided multipath can easily be transformed into disjoint multipath. As shown in Figure 3, there exists four multipath between the source and destination and three of them are overlaid. The data splitting algorithm uses only the failing probability of each path. This means that we can consider the three paths from source to node $\mathrm{X}$ as a separate disjoint multipath problem, apply the algorithm presented in the next section to it and get a failing probability for it. This way we can reduce the group of braided paths to a single path. It is easy to see that the simple example presented here can be generalized for any type of configuration.

By this process we get multiple paths from source to destination. Let there is $\mathrm{K}$ paths from sink to source. That time we divide the full packet into $\mathrm{k}$ sub packets and send them via $\mathrm{K}$ multiple paths. Then they try to discover how many sub packets are successfully send to sink. Those sub packets which are missing the sending time are need to retransmitted. Here every node between source and sink has a failing probability. Failing probability is calculated from its previous ability of successfully sending data from source to sink. From the failing probability they calculate Ek. This gives us the number of successful paths among all multiple paths that are able to successfully send data from source to sink. We calculate Ek from the failing of the nodes for every path. Upper bound of failing probability is 0.25 . If there are $\mathrm{k}$ sub packets are available in the network and sink receives Ek sub packets successfully then they need (k-Ek) sub packets to reconstruct the original packet.

\subsection{Energy-Efficient Multi-Path Routing Protocol}

Two main benefits can be achieved by multi-path routing: First, as the transmission load is balanced among multiple paths the energy of nodes is burned more equitably. Thus, energyefficiency is increased and the network lifetime is prolonged. Second, multiple paths provide redundancy for data transmission, which increases the reliability of delivery. In this paper, a novel multi-path routing protocol in WSNs is proposed, which can discover multiple paths with high energy efficiency. There proposed protocol[4] is composed of three phases: double routing trees construction, route discovery and data transmission. 
This protocol constructs an energy efficient tree. This tree is known as double routing tree. In this double routing tree at first we try to find shared node. Shared nodes are that nodes which are connected with both sink node and source node. We get different path from source to sink via shared node. From shared node we find successful path from source to sink. Then using this routing paths data is delivered from sink to source node

Double routing trees construction phase constructs two trees: query tree which is rooted at sink node and search tree which is rooted at source node. The construction of two trees begins and ends at the same time. The period of construction is pre-assigned, which determines the size of two trees, i.e. the number of nodes in two trees. After two trees have been constructed, there are some nodes which belong to both of two trees, called shared nodes. The longer the construction period of two trees is, the more the shared nodes are. By this way we determine the shared nodes.

\section{DATA SPlitTINg TECHNIQUE}

Let, there is $\mathrm{K}$ multiple paths[3] from source to sink node. That time we must split the original packet in $\mathrm{K}$ number sub packets of equal size. These $\mathrm{K}$ sub packets are sending through different $\mathrm{K}$ multiple paths from source. In sink we need all $\mathrm{K}$ sub packets for reconstruct the original message. If we get all $\mathrm{K}$ sub packets in sink then we can successfully reconstruct the original message. But if we receive less than $\mathrm{K}$ sub packets in sink that time need to retransmit the missing sub packets from source to sink. After receiving the retransmitted packets we can able to fully construct our original message in sink node. The data splitting technique is briefly discussed below:

Suppose we want to send a data packet from a source to a destination and the process of route construction is finished, resulting in $\mathrm{k}$ different paths that are to be used. Each path has some rate $\mathrm{pi}(\mathrm{i}=1, \ldots, \mathrm{k})$ that corresponds to the probability of successfully delivering a message to the destination.

This setting corresponds to a repeated Bernoulli experiment, the i-th subrun corresponding to the message transmission along the $\mathrm{i}$-th path. Note that we consider node-disjoint paths and therefore can assume these experiments to be independent of each other. Let Sk: $\{0,1\} \mathrm{k} \rightarrow \mathrm{N}$ be the random variable corresponding to the number of successfully delivering paths.(For Sk, each subrun is assigned a 1 if the transmission was a success along the respective path, and 0 if it failed. Then, Sk represents the sum of these for the subruns, and clearly Sk k.) Then, the expectation for the total number of successful paths is given by

$$
\mathrm{Ek}=\max \left\{\mathrm{Xu} \sqrt{\sum_{i=:}^{k} \mathrm{Pi}(1-\mathrm{Pi})}+\sum_{i=1}^{k} \mathrm{Pi}\right\}
$$

This Ek returns successful path. Then from here we found lost packet numbers and resend them through different paths. And regain all packets in destination node. This splitting technique reduces traffic overhead and network congestion in the network significantly. This data splitting technique in multi path routing protocol is a revolutionary process.

\section{OUR Proposed TechniQue}

In a wireless sensor network, communication among the sensor nodes depends on reliable path from a source node to a sink node. Also there requires reduced traffic to send data packet efficiently. For this purpose we propose a combine technique to ensure both reliability and 
reduced traffic in network paths. The technique combines well known energy efficient multi-path routing protocol for generating energy efficient multiple reliable paths from source to destination and data splitting technique for reducing traffic overhead among these multiple paths. Both the techniques are describe below.

\subsection{Energy Efficient Routing Tree Construction}

Every node of a wireless sensor network contains very limited energy and bandwidth resources, high density of node deployment, and cheap and unreliable sensor nodes which are prone to failures. For this reason it is very necessary to route from search node to sink node energy efficiently. In our implemented algorithm we try our best to route energy efficiently.

There are three kinds of nodes in the wireless sensor network. They are source node, sink node, and normal node. Source nodes are those node which start to transmit the data. Sink node are those node which are the destination of data. And the data are transmitted through the source node to sink node via normal node. Here we use two kinds of message. One is sink message and other is search message. The message that is sent by sink node and is to find search messages is called sink message. The message that is sent by source node and is to find query messages is called search message. We construct two trees, one is query tree and another is search tree. The construction of two trees begins and ends at the same time. After two trees have been constructed, there are some nodes which belong to both of two trees, called shared nodes.

We use a local register for each node called register 1 which is shown in Figure 1.The register contains 5 field. These are level, father id, query, search, state. When we construct search and query tree the register is used. The structure of the search and query message is shown in Figure 2. Now we are going to construct query tree first, one node of the network is interested in some sensing data and becomes sink node. It enters query tree to become the root of the tree, and assign itself level 0 . Then, sink node broadcasts query messages. In a query message, the level and ID of the node from which the message is sent are recorded. Another pre assigned parameter is Threshold field which represents the threshold of residual energy in the nodes. Every neighbor of sink node receives a query message sent from sink node. It records the ID of sink node, assigns itself a level which is one greater than the Level of the message and sets the Query field to 1 . Thus, the node enters query tree and becomes a child of sink node. Then, the node compares its residual energy value and the Threshold field of the message. If the former is larger than the latter, it adds 1 to the $\mathrm{Nm}$ field of the message. Contrarily, it adds 1 to the $\mathrm{Nl}$ field of the message. After that, the node checks the Search field of its local register 1. If the Search field is 0, it acts as follows: I. Compares the Nm and Nl. If Nm is larger than Nl, it sets the State field to 1; II. Broadcasts the query messages with new Level, Father ID and Nm or Nl to its neighbors. If the Search field is 1, i.e. the node has entered search tree and becomes a shared node, it compares the $\mathrm{Nm}$ and $\mathrm{Nl}$. If $\mathrm{Nm}$ is less than $\mathrm{Nl}$, it multiplies the State field by 0. The above process continues. What should be noticed is that a node which has entered the query tree can receive a query message again. When this happens, the node neglects any such messages.

\begin{tabular}{|l|l|l|l|l|}
\hline Level & Father ID & query & search & State \\
\hline
\end{tabular}

Figure 1. The structure of local register 1

\begin{tabular}{|l|l|l|l|l|}
\hline Level & Father ID & Thershold & Nm & NI \\
\hline
\end{tabular}

Figure 2. The structure of query, search messages 
Construction of search tree is also the same like as the query tree. To construct search tree first, one node of the network is interested in some broadcasting data and becomes source node. It enters search tree to become the root of the tree, and assign itself level 0 . Then, search node broadcasts search messages. In a search message, the level and ID of he node from which the message is sent are recorded. Building of search tree now follows the same procedure as query tree.

\subsection{Applying Efficient Data Splitting Technique}

After constructing the energy efficient double routing tree we get the shared nodes. By checking the state field of each node of the sensor network we find the path which has no chance of failure. So we get all the active paths from source to sink. Then we use this active paths for sending data. These active paths never failed in sending data because we use threshold value and residual energy all of the nodes of the network. The nodes which are in the active path must have the ability of forwarding message to the next node. So there is no chance of data failure. We take this advantage of no data failures and use data splitting technique.

Say we discover K multiple paths from source to sink. This all are active path. There is no chance of data failure in these paths. Now we split our total data packet into k sub packets. We send this $\mathrm{k}$ sub packets through $\mathrm{k}$ distinct path. Here all paths are guaranteed. So in sink we get all these $\mathrm{k}$ sub packets. Now in sink we combine k sub packets together and get our original packet. Now we describe this data splitting technique with an example in below.

Let we want to send a packet of size 10 unit from source to sink node. And there are many other nodes between source and sink. Using the process of double routing tree construction, we discover that there are 4 activated paths from source to sink for data transmission. Then we split the packet size of 10 unit data in to 4 sub packets. Each sub packets contain 2.5 units of data. Using the multiple paths these data reached to sink node successfully. Then we combine 4 sub packets together and recover full packet efficiently.

\subsection{Reducing Traffic Overhead and Ensuring Reliability}

In our designed protocol we use such a double routing tree where is no chance of failing any data in the network. So when we apply our data splitting technique that time there is no chance of retransmission of data or missing of any packet.

If we use no data splitting technique in our algorithm then we send same packet through multiple path. Let if we do not use data splitting technique and send full packet through multiple path. The traffic on the network increases exponentially. Let here we want to send 10 unit data and there is 5 multipath. In this case we send full packet through all the paths. We in sink node we receive data from any of the path. In this case total traffic is 50 because there 50 unit data in the network.

In another existing paper[3] they use data splitting technique but their paths are not guaranteed. For this reason they need to data retransmission. For this reason traffic also increases. Let 10 unit data send through 5 multiple path. Each path contains 2 unit of data. If one path failed then we again send the 2 unit data. So from source to sink there traffic is 12 .

But in our data splitting technique we get better output than previous two examples. Here let the same situation as 10 unit data and 5 multiple paths. In our algorithm all paths are guaranteed. So here is no data missing in the network. For this reason when we send sub packets we receive all this in the sink node. So total traffic is 10 . From the above discussion we can see that our proposed algorithm reduced traffic overhead efficiently. 
In our proposed protocol when we send data from source node to sink node at first we ensure the path. From all the paths we select only those paths which are the ability of forwarding message or data to the next node. So we say that our proposed protocol works with only successful path.

But in previous existing routing protocol [3] we see they not use guaranteed path. They select the path on the basis of failing probability of node. They calculate the failing probability of node on the basis of previous experience. So there is some sub packet is missed. So the previous protocol does not ensure reliability. On the other hand our designed protocol has no chance of data packet failure. From the above we can say that our protocol ensure reliability.

\section{SiMULATION AND RESULTS}

In this section we discuss about simulation process and outcomes of the simulations. We use $\mathrm{C} / \mathrm{C}++$ to simulate our code. We store the output of our simulation code on a text file. From output data we try to establish relationship among different parameters such as multi path degree, traffic, failing probability, failed transmission etc.

In Figure 4 we establish relationship between multipath degree and traffic overhead. Here is the effect of failing probability. In this case all path is activated. Here they use no data splitting. We implement the algorithm in [4] and plot the output data in a graph plotter and get this relationship. Here in simulation we send a packet size of 10 unit from source to sink. In their algorithm they said that send the full packet in every path. There is no chance of data failing in any path. So for this reason with the increase of multi path degree traffic increases linearly.

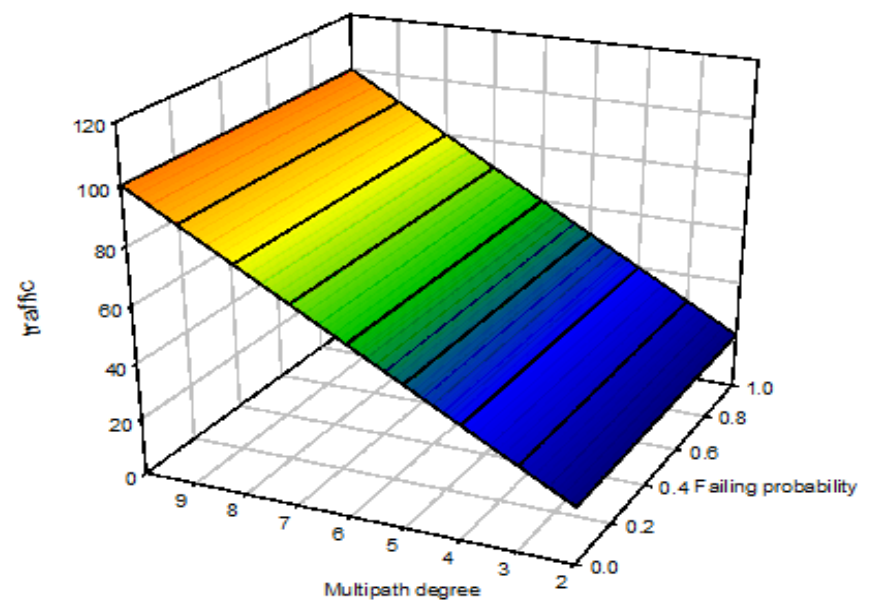

Figure 4: Traffic vs. Multipath degree in energy efficient routing protocol when packet size is 10 unit

We increases the size of the packet from 10 unit to 15 unit. Then we apply this to their algorithm we observed a relationship between multipath degree and traffic overhead show in Figure 5. We see that here also the traffic increases linearly with the increase of multipath degree. Furthermore we observe from Figure 4 and 5 that increasing the packet size increases the traffic overhead. 


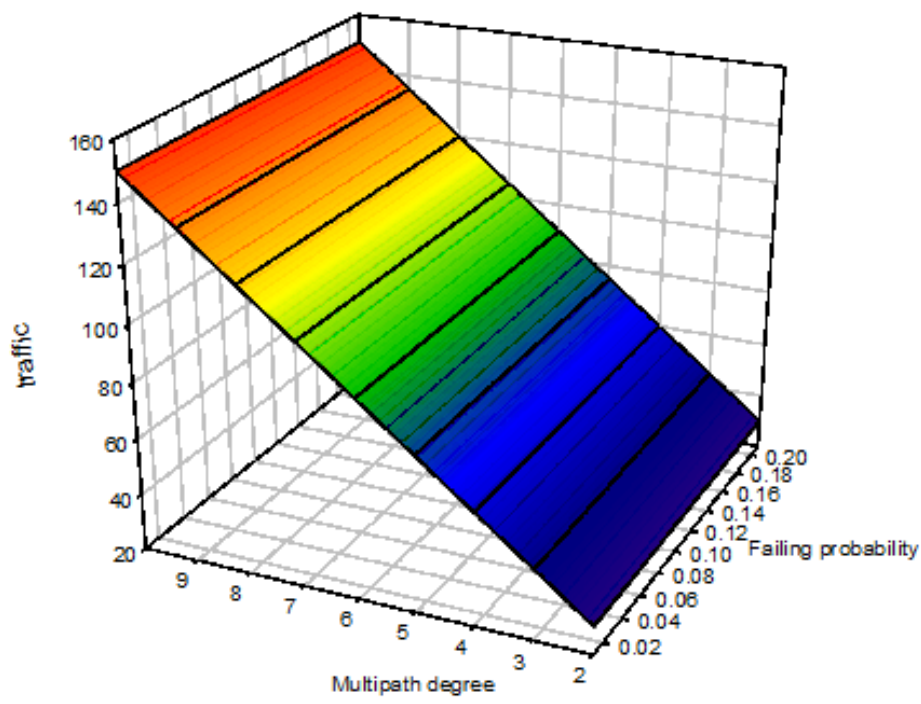

Figure 5: Traffic vs. Multipath degree in energy efficient routing protocol when packet size is 15 unit

When we implement our own proposed algorithm we get a better feedback between Traffic and multipath degree. Here we use same input as above two figures. In our implemented algorithm we see that it contains less traffic overhead in the network than previous existing routing protocol.

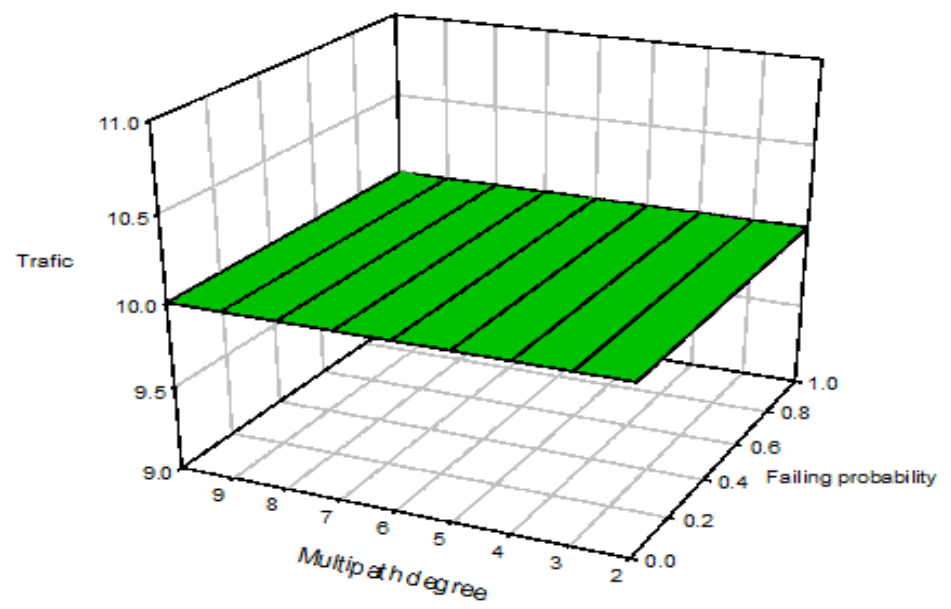

Figure 6: Traffic vs. Multipath degree in our proposed protocol when packet size is 10 unit

In Figure 6 we observe that always with the increase of multipath degree, traffic does not increased. Here is no effect of failing probability. Because here is no chance of failing data in any node and thus here is no retransmission in our algorithm. And the main cause is efficient data splitting technique. In figure 4 and 5 they support reliable path and no need of retransmission but they not use good data splitting technique. 


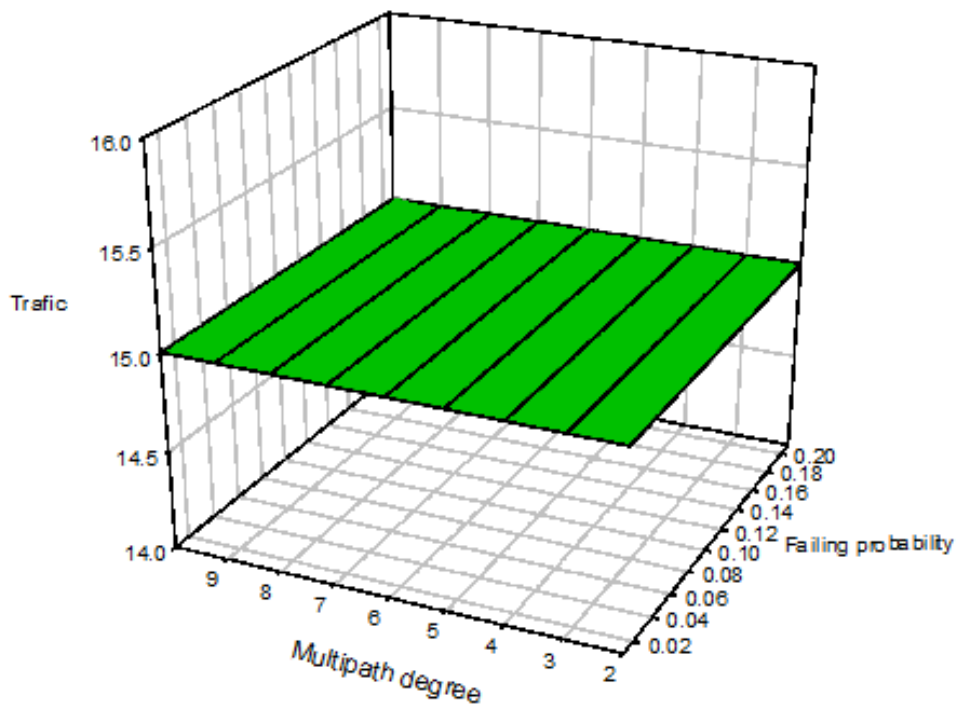

Figure 7: Traffic vs. Multipath degree in our proposed protocol when packet size is 15 unit

But in our algorithm we use both properties. We use reliable path and good data splitting technique. For this reason if we send 10 unit data from source to sink traffic is always 10 unit. Also we can see from the Figure 7 that increasing data packet from 10 to 15 unit does not cause the change in overall traffic and remain constant for different multipath degree. This is our main achievement over previous two existing algorithm.

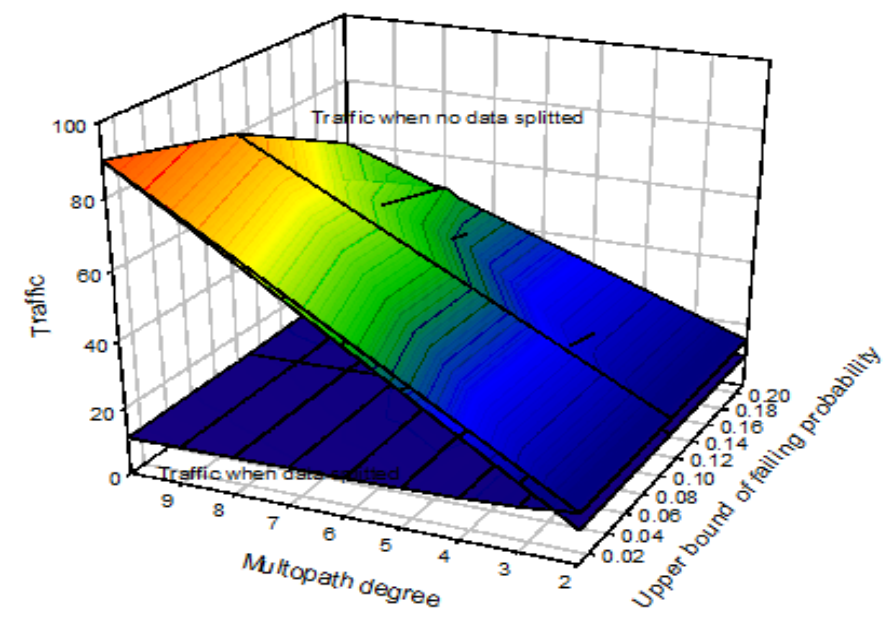

Figure 8: Data splitting vs. no data splitting when packet size is 10 unit.

In Figure 8 we establish a relationship among multipath degree, traffic and failing probability. There are two plane one for data splitting and another for without data splitting. In case of without data splitting with the increase of multi path degree traffic on the network increases linearly. But when we use data splitting technique traffic remains constant with increase of multipath degree. 
International Journal of Computer Science \& Information Technology (IJCSIT) Vol 4, No 3, June 2012

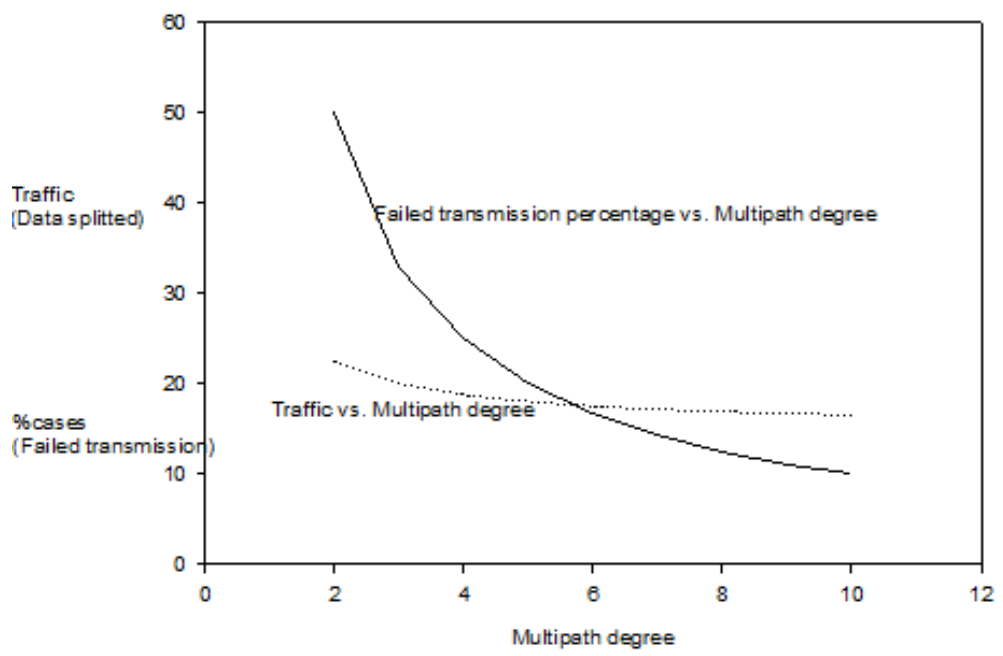

Figure 9: Relation of traffic and failed transmission with multipath degree when packet size of 15 unit

In Figure 9 comparison occurs between failed transmission versus Multipath degree and Traffic versus multipath degree in data splitting technique proposed in [3]. Here we use packet size of 15 unit. In this case failed transmission decreases exponentially with the increase of multipath degree. On the other hand the traffic remains almost the same for different multipath degree with a slight increase at initial stage.

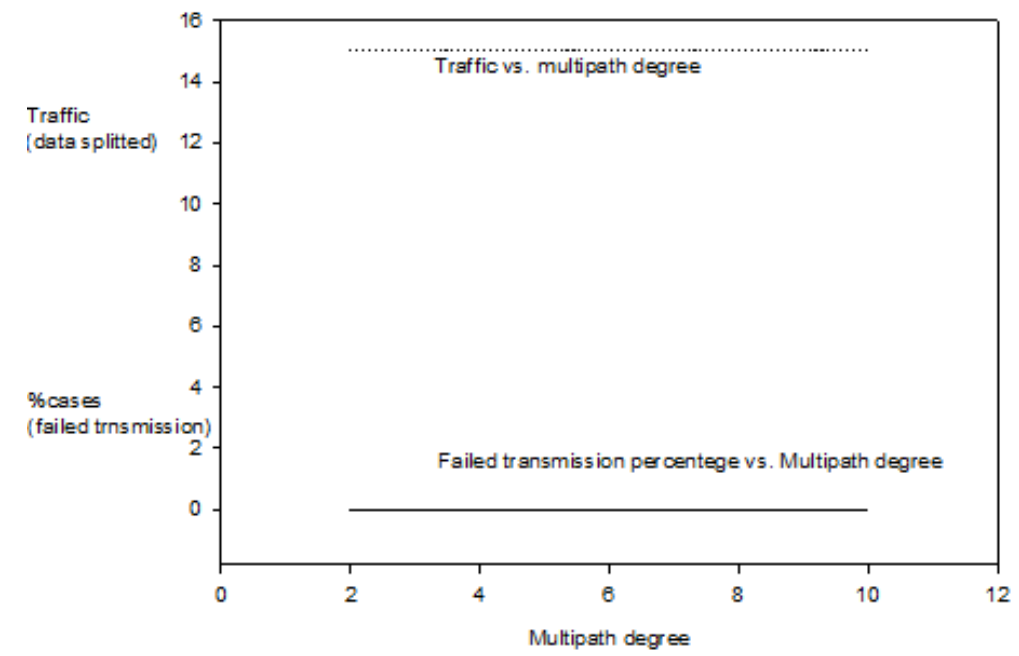

Figure 10: Relation of traffic and failed transmission with multipath degree in our proposed protocol when packet size of 15 unit

By implementing and simulating our own protocol in figure 10 we get a relation between failed transmission vs. Multipath degree and Traffic vs. multipath degree. Here we use packet size of 15 unit. Here failed transmission is always zero because in our algorithm at first we ensure the path and then send data. For this reason always our failed transmission is zero. On the other hand traffic is always same for same size packet. For a same size packet traffic is not change with increase or decrease of multipath degree. This is our revolution with compare to the previous existing algorithm. 


\section{Conclusions}

To improve the reliability of the wireless sensor network the data packet must be transmit in different path. A multipath routing protocol provides the facility to transmit the data packet in different path. On the other hand if same the data packet is sent in different path the network becomes congested. So it is necessary to provide such an algorithm which will provide the reliability and decrease the number of packets in the network. There is another problem of transmitting data in different path. This problem is all paths of the network is not working well. As a result if we transmit the data packet in all the paths without verification then there may be a problem of data loss. So these are all the fact of transmitting data from source node to ink node. Our implemented algorithm fulfills all kinds of requirement that is need for reliability and decrease the network congestion. In our algorithm first we discover the path which are work well in transmitting data from source node to sink node. Then we split the data into k sub packet where $\mathrm{k}$ is the number of multipath which are work well in transmitting data from source node to sink node. Thus we ensure the reliability and decrease the number of packets from the network.

In sensor network there is a limitation of power. If a protocol is designed such a way that needs more power to transmit data from source node to sink node then it will not be realistic. we design our protocol is considered this condition while design our protocol.

we implement and simulate our proposed protocol. We also implement the protocol which seems to more relate to ours one. Then we compare the simulation output of our implemented protocol with reference protocols. The comparison between of our implemented protocol and the reference protocol are given above. From this comparison it is clear that our protocol is better than other existing protocol in the sense of energy efficiency, reliability and decrease the traffic overhead.

\section{REFERENCES}

[1] Jamal N. Al-Karaki Ahmed E. Kamal, "Routing Techniques in Wireless Sensor Networks: A Survey”Dept. of Electrical and Computer Engineering Iowa State University, Ames, Iowa 50011.

[2] Stefan Dulman, Tim Nieberg, Jian Wu, Paul Havinga,"Trade-Off between Traffic Overhead and Reliability in Multipath Routing for Wireless Sensor Networks,'Faculty of EEMCS,University of Twente,Enschede, the Netherlands

[3] Sachin Sharma, Dharmendra Kumar and Rajesh Kumar,'QOS-Based Routing Protocol in WSN'Department of Electronics \& communication, KIET, Ghaziabad, India;Department of Physics, SMIT, Sikkim, India

[4] Ke Guan, Li-Ming He, “ A Novel Energy-Efficient Multi-Path Routing Protocol for Wireless Sensor Networks," School of Information Engineering Chang'an University, Xi'an, China, ,School of Information Engineering, Chang'an University, Xi'an.

[5] Li-Ming He, “ A Novel Multi-Path Routing Protocol in Wireless Sensor Networks," School of Information Engineering, Chang'an University, Xi'an.

[6] Deepak Ganesan, Ramesh Govindan, Scott Shenker, Deborah Estrin, "Highly-Resilient, EnergyEfcient Multipath Routing in Wireless Sensor Networks"Computer Science Department, UCLA,International Computer Science Institute, Berkeley,ACIRI, Berkeley.

[7] He Huang, Yun Xu, Yu-e Sun, Liusheng Huang”Cluster-Based Load Balancing Multi-path RoutingProtocol in Wireless Sensor Networks”Department of Computer Science and Technology,Shenyang Institute of Computing Technology,University of Science and Technology of China ,Chinese Academy of Science,Hefei, 230027, Anhui Province, China Shenyang, 110004, Liaoning Province, China

[8] Peng Yang,Biao Huang,"Multi-path Routing Protocol for Mobile Ad Hoc Network"Chongqing University of Arts and Science Chongqing, China,Chongqing University of Arts and Science Chongqing, China. 
[9] Lu Xuechao, Xu Chunxiu, Wu Muqing, Zhen Yan, Wu Dapeng,'Design and Realization of a Novel Multi-path Load-Balancing Routing Protocol in Ad Hoc Network"Broadband Communication Network Lab Beijing University of Posts and Telecommunications Beijing, China.

[10] Li Ming, Huang Changlai, Yang Jianghu, Peng Gegang, Gao Chuanshan,’Multi-path QoS Routing Protocol under Three Different Path Selection Strategies in Wireless Ad-Hoc Networks"Department of Computer Science and Engineering, Fudan University 220 Handan Rd. Shanghai, PRC 200433.

[11] Peter Pham and Sylvie Perreau,"Multi-Path Routing Protocol with Load Balancing Policy in Mobile Ad Hoc NetworkUniversity of South Australia, Mawson Lakes Blvd, Mawson Lakes. SA5059, Australia"

[12] ChristosTachtatzis, DavidHarle "PerformanceEvaluationofMulti-pathand SinglepathRoutingProtocolsfor MobileAd-HocNetworks"Department of Electronic \& ElectricalEngineering,UniversityofStrathclyde,Glasgow,G11XW,Scotland,UK

[13] Rahul C. Shah and Jan M. Rabaey"Energy Aware Routing for Low Energy Ad Hoc Sensor Networks"Berkeley Wireless Research Center,University of California, Berkeley.

\section{Authors}

G.M. Mashrur-E-Elahi received the B.Sc. Engg. degree (with honors) in computer science \& engineering from Khulna University of Engineering \& Technology, Khulna - 9203, Bangladesh, in 2009. He is currently working as a Lecturer in the same department of this university. He has published one International Journal and two International Conference papers. His current research interests include Computer and wireless mobile networks, Evolutionary computing, and Computer algorithms. Mr. Mashrur is an Associate Member of the Institute of Engineers Bangladesh (IEB).

Din Mohammad Mobin received the B.Sc. Engg. Degree in computer science \& engineering from Khulna University of Engineering \& Technology, Khulna - 9203, Bangladesh, in 2011. He is currently working as a freelance web developer. His current research interests include wireless mobile net works, and routing protocols in wireless sensor network

Md. Anwar UI Islam Rajib received the B.Sc. Engg. degree in computer science \& engineering from Khulna University of Engineering \& Technology, Khulna - 9203, Bangladesh, in 2011. He is currently working as a Sr. Software Engineer in Flamma Corporation Ltd Bangladesh. His current $r$ esearch interests include wireless mobile networks, and routing protocols in wireless sensor network
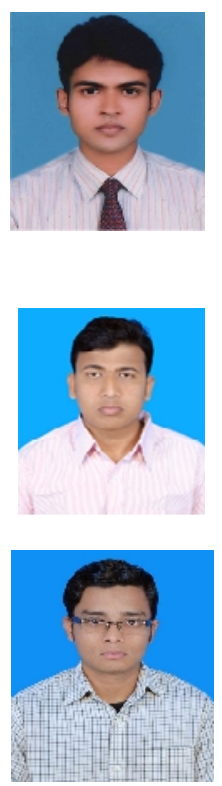